\section{Bei Kopf-Hals-Tumoren bringt Epo nichts}

Patienten mit Anämie sprechen meist schlechter auf eine Strahlentherapie an als Patienten mit normalen Hämoglobinwerten. Das gilt auch bei Kopf-Hals-Tumoren. Eine logische Schlussfolgerung wäre, dass die Behandlung der Anämie mit Erythropoetinen den Therapieerfolg verbessern könnte.

D ie Wirkung von Epoetin alpha auf das Überleben und eine behandlungsbedingte Anämie und Fatigue wurde an 301 Patienten mit Plattenepithelkarzinomen an Kopf oder Hals untersucht. Alle Patienten wiesen Hämoglobinspiegel unter $15 \mathrm{~g} / \mathrm{dl}$ auf und unterzogen sich einer Radiotherapie (RT) mit kurativer Absicht entweder allein oder mit zusätzlicher Gabe von Epoetin alpha (10.000 bzw. 4.000 Einheiten bei einem Hb-Wert < bzw. $\geq 12,5 \mathrm{~g} / \mathrm{dl}$, s. c., dreimal pro Woche). Der primäre Endpunkt war das lokalrezidivfreie Überleben (DFS).
Die Hb-Spiegel stiegen unter Epoetin alpha im Verlauf auf 13,65-14,87 g/dl. Aber beim DFS gab es keinen signifikanten Unterschied zwischen beiden Gruppen (35,42 Monate unter alleiniger RT bzw. 31,47 Monate unter RT plus Epoetin alpha; $\mathrm{p}=0,791) .43,9 \%$ bzw. 41,3\% der Patienten lebten mindestens fünf Jahre ohne Lokalrezidiv. Die Zeit bis zum Lokalrezidiv und das Gesamtüberleben (58,64 bzw. 56,67 Monate; $\mathrm{p}=0,823)$ waren ebenfalls vergleichbar. Darüber hinaus sprachen die Tumoren vergleichbar gut auf die Radiotherapie an. In beiden Gruppen erreichten nach zwölf Monaten $99 \%$ der Patienten eine komplette oder partielle Response im Primärtumor, 96\% in den Lymphknoten.

Keine Unterschiede zeigten sich auch in der behandlungsbedingten Anämie oder Fatigue, gemessen anhand des FACT-Anemia und des FACT-Head and Neck. Neue oder unerwartete unerwünschte Ereignisse traten nicht auf.

Fazit: Die Behandlung mit Epoetin alpha zusätzlich zur Radiotherapie hat bei Patienten mit Kopf-Hals-Tumoren keinen Einfluss auf Überleben, Tumor-Response, Anämie und Fatigue. Das bestätigt die Ergebnisse früherer Studien. jn

Hoskin PJ et al. Effect of epoetin alfa on survival and cancer treatment-related anemia and fatigue in patients receiving radical radiotherapy with curative intent for head and neck cancer. J Clin Oncol 2009; 27: 5751-6. 